\title{
High-Level Coordination of Business Processes in a Virtual Enterprise
}

\author{
Luiz M. Spinosa, Ricardo J. Rabelo, Alexandra P. Klen
}

G-SIGMA -Intelligent Manufacturing Systems Group Federal University of Santa Catarina / Mechanical Engineering Department / Grucon

Telephone (+55 48) 3319387 Telefax (+ 55 48) 2341519 http://www.gsigma-grucon.ufsc.br 88040-900 - Florianópolis (SC) - Brazil P.O.Box 476 E-mail: \{spinosa,rabelo,aklen\}@gsigma-grucon.ufsc.br

\begin{abstract}
In a Virtual Enterprise scenario the management of the supply-chain is a complex task, especially when some degrees of flexibility are envisaged to be supported. This paper intends to show an approach to improve the quality of this management in order to avoid business chaos. More preciselly, it is introduced a set of specialized software modules named Advanced Coordination Functionalities (ACFs), which take end-user feedback into account to solve important problems during coordination actions within the VE scenario. The study of ACFs is motivated and required by the Prodnet-II ESPRIT project.
\end{abstract}

\section{Keywords \\ Coordination, Distributed Business Processes, Virtual Enterprise}

\section{INTRODUCTION}

The virtual enterprise (VE) concept is a predominant area of research and technological development nowadays. The essential assumption is that manufacturers do not produce complete products in isolated facilities, but rather 
they operate as nodes in a network of suppliers, customers, engineers and other specialized services (Hunt \&al., 1997). VEs materialize themselves by selecting skills and assets from different firms and synthesizing them into a single business entity, the so-called 'value-chain' (Bowersox \&al., 1996).

The VE must also deal with orders requests. In this case, these orders are named distributed business processes (DBP). A DBP is a dynamic and temporary set of business processes (BP) which jointly gives rise to the end product of the VE. As the BPs are supposed to be performed by several enterprises, the enterprise that triggered the formation of a given VE normally must coordinate their execution in order to avoid business chaos (Rabelo \&al., 1996). In a VE scenario the management of the value-chain is a complex task, especially when some degrees of co-ordination are envisaged to be supported.

The work to be presented in this paper corresponds to the approach being implemented in the Prodnet-II (Camarinha-Matos \&al., 1997) ${ }^{1}$ to support that coordination. Prodnet-II aims to design and develop an open platform and the adequate IT protocols and mechanisms to support the virtual industrial enterprises. It focuses on Small and Medium size Enterprises in order to support them with means to inter-operate with several value chain networks.

\section{HIGH-LEVEL COORDINATION REQUIREMENTS}

The full set of high-level coordination activities in the VE should comprise the entire VE life cycle, i.e. the phases of VE creation, operation and dissolution. Therefore, the requirements to high-level coordination emerge from the analysis of activities within each of these VE phases. In this sense, a non-exhaustive list of such activities is described below:

\section{a) VE Creation}

General phase aiming to form a group of enterprises to accomplish a certain business process (BP). Since the BP is to be carried out by a set of enterprises, namely the nodes of the network, it becomes a DBP. Each node could be responsible for one or more BPs. The execution of a DBP represents the concrete and essential goal of a VE.

\section{1 - VE formation}

General activity which aims to find a logical consortium of enterprises.

\footnotetext{
${ }^{1}$ Prodnet-II is a research project partially supported by the European Union (ESPRIT project 22647) and by the CNPq (The Brazilian Council for Research, project 680120/96.3). It involves the following partners: CSIN (PT), New University of Lisbon (PT), ESTEC (PT), Miralago (PT), University of Amsterdam (NL), Lichen Informatique (FR), CIMIO (UK), Federal University of Santa Catarina (BR) and Herten (BR).
} 


\section{1 - DBP announcement elaboration}

Edition of the announcement associated with the DBP specifications (information related with technical aspects, quality, monitoring, production, etc.) that goes to be spread in the network.

\section{2 - Seeking potential partners}

Searching enterprises that match the DBP requirements indicated in the announcement.

\section{3 - Reception of proposals / selection of partners}

Managing the reception of proposals (bids) for each DBP announced and further selection of the appropriate partners for the DBP's.

\subsection{1 - Negotiation}

A negotiation process may be established when conflicts take place during the selection of partners (Rabelo \&al., 1994). This negotiation can also occur during the operation phase.

\subsection{2 - Commitment}

Final agreement about a certain DBP.

\subsection{3 - Final result spreading}

The bidders are communicated about the final result.

\section{4 - DBP material and data inter-flow planning}

The distributed workload has to be planned, especially for logistics issues (Ross, 1996).

\section{5 - Legal agreement}

The VE concept does not replace the necessity to legalize the agreements reached. Hence, the DBP's contract has to be duly signed and legalized, which may implicate in some incompatibilities between the laws applied on different geographical areas in which the VE's nodes (VE members) are placed.

\section{2 - VE instantiation}

Once the logical consortium of enterprises is met the VE has to be properly «instantiated». The consortium formed to compose a VE is seen as an «instance» of a VE concept. Once an instance is created a general set up is required for all of the VE members so that the VE can adequately run.

\section{b) VE Operation}

General phase that covers the actions performed by a VE during its existence.

\section{1 - DBP management}

Activity that comprises the normal execution of a DBP.

\section{1 - DBP process evaluation / measurement / simulation}

A DBP needs to be constantly checked in order to guarantee that the VE goal shall be achieved. This checking could be made from the following perspectives:

1.1.1 - Technical (production and logistics aspects)

1.1.2 - Social / human impacts 


\subsection{3 - Legal}

\section{2 - DBP monitoring}

A basic functionality for the VE management consists in getting information and querying about a certain DBP and/or VE members (the suppliers) as well as allowing it to be visualized.

\section{2 - $\underline{\text { Supervision }}$}

Activity especially devoted to deal with any disturbance in the DBP management (Rabelo \&al., 1997).

\section{1 - VE Reconfiguration}

A VE may have to be reconfigured according to a disturbance severity. In some cases it can provoke the need for a rescheduling at the VE level.

\subsection{1 - Inclusion of new member}

It is related with the introduction of a new enterprise in the VE (i.e. during the execution of a certain DBP) because a VE member failed and its replacement is needed or due to some modifications in the DBP which may require the contracting of a new enterprise.

\subsection{2 - Exiting of a member}

It is related with the exiting of a VE member due to a disruption caused by its own failure or to some modifications / cancellation / renegotiations / in the DBP contract.

\section{2 - Modification on the DBP contract}

The DBP may have its specifications changed along the VE execution, such as the due date, some technical specifications, transport procedures, etc..

2.2.1 - Ordered by the VE coordinator

A modification in a DBP may be triggered by the VE coordinator by means of a DBP re-announcement and/or re-planning.

2.2.1.1 - Due to a VE member failure

That modification can be originated from a VE member that has failed which obliges the VE coordinator redo the DBP announcement aiming at its accomplishment.

2.2.1.2 - Changes in the DBP contract impose a VE configuration

The VE coordinator may change a certain DBP specifications that may oblige the VE reconfiguration.

\subsection{2 - Requested by one VE member}

The own VE member may request a DBP modification to the VE coordinator due to some particular reason.

\section{3 - Conflict evaluation}

A conflict occurs whenever any problem concerned to a DBP takes place that offends the current contract. The decision-making process can be very complex, depending on the nature of DBP, the number and type of partners and technological processes involved, the costs evaluation, etc.. A global evaluation upon a conflict provides an enterprise means for a better decision-making (Spinosa \&al., 1997). 


\section{c) VE Dissolution}

\section{1 - Normal dissolution}

Once the DBP execution is finished the VE ends. The VE dissolution happens when each VE member performs its BP(s) correctly, according to the contract.

\section{2 - Problematic dissolution}

This dissolution occurs when a DBP is not successfully completed. It happens when a conflict arises and cannot be solved.

\section{ADVANCED COORDINATION FUNCTIONALITIES}

Advanced Coordination Functionalities (ACF) are high-level services, which «implement» the VE coordination activities. ACFs correspond to specialized software modules being developed to help solving specific problems improving the quality of the management of the supply-chain. The ACFs are integrated within the Prodnet-II Platform, which corresponds to an upper software layer to be installed on the enterprises' legacy systems (their internal modules) so that the enterprises can operate in a VE scenario in a «transparent» and integrated way. In fact, two main modules constitute this platform: the cooperation layer and the ACF module (figure 1).

The cooperation layer comprises a number of semi-autonomous software modules that provides the enterprise with several other functionalities, such as: exchange of commercial data (using EDI/EDIFACT and WWW), exchange of technical product data (STEP), quality related information exchange, VE information management, the management of incomplete and imprecise orders. All these «services» are supported by other basic software modules to guarantee the privacy and safety communication, distributed data management, architecture/services configuration and global coordination among the modules. A Communication Protocol supports the communication between the ACFs and the cooperation layer, as well as with abroad (Camarinha-Matos \&al., 1997).

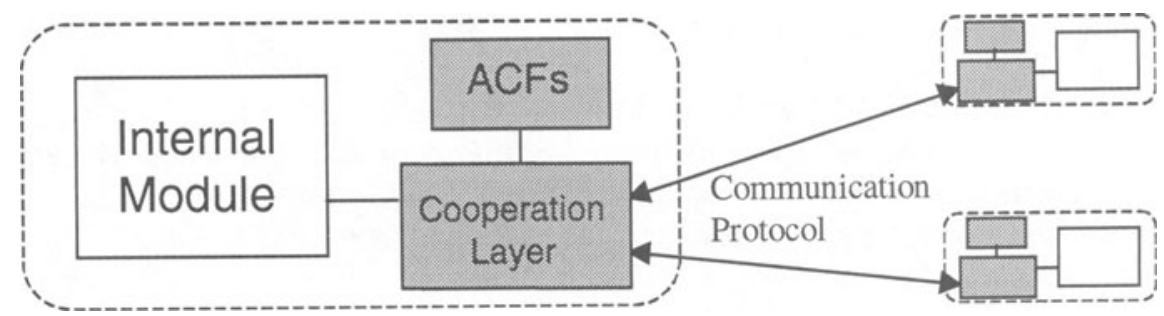

Figure 1 - Prodnet-II General Approach.

One can note that there are several ACFs that can be designed to help an enterprise in the high-level coordination of a VE. The five ACFs introduced below correspond to the ones being researched within the Prodnet-II project (Figure 2). 


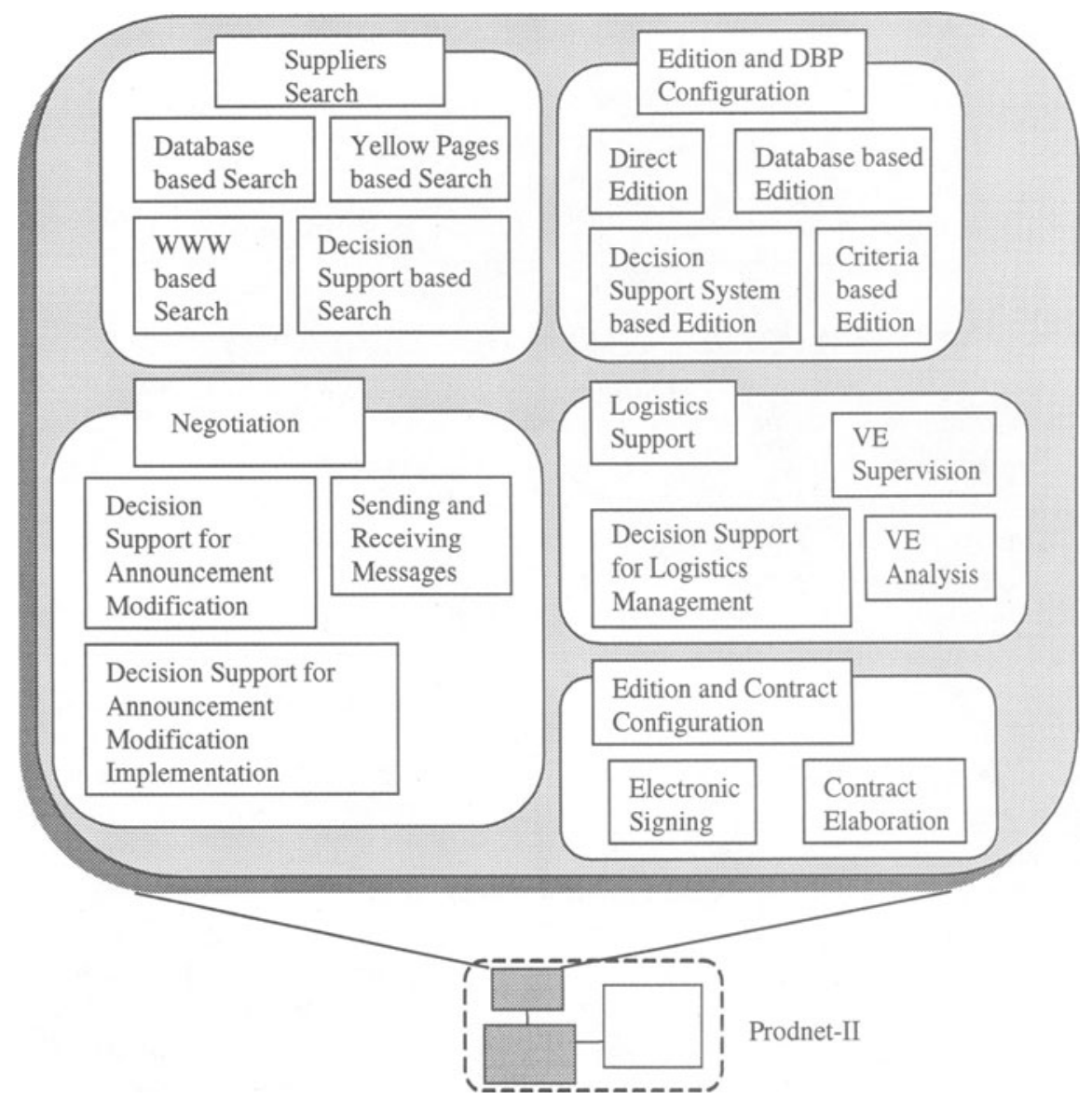

Figure 2 - The ACFs General Architecture

\subsection{Edition and DBP Configuration}

This ACF matches the sub-activities 1.1 (DBP definition and announcement elaboration) and 2.2 (modification in the DBP contract), identified in the Chapter 2. It aims at supporting a computer-aided edition and configuration of the announcement associated with a DBP that goes to be spread in the network in order to look for enterprises interested and capable to attend (part of) it. An announcement is a complete information model through which an enterprise specifies their necessities and requirements that the suppliers must comply with. An announcement normally contains due dates, costs proposals, ways of payment, technical information, access rights, information to be remotely monitored, etc., that can vary from industrial domain to industrial domain. In a VE environment, the elaboration of an announcement - and its further sending through the network - 
is the trigger event for a VE creation. This activity can be executed in four different ways (but not mutually exclusive):

- Direct Edition. In this simplest way of editing, the user fills an information structure directly on a file or via a specific graphical interface, indicating the announcement's values. Because the nature of the attributes can be quite variable, different departments of the enterprise can fill an announcement. It means the need for a distributed software plataform in order the departments can fill the announcement in a cooperative and synchronized way.

- Database based Edition. An announcement can be completely filled when receiving right data from a database that contains a list of typical announcements, which can be searched by keywords, for instance.

- Criteria based Edition. This way is similar to the previous one. Here, the search would be made based on «qualitative» values, such as «competence factors». For example, in some cases an enteprise is not interested to find partners able to produce a certain piece, but also that use to be very responsesensitive. It means the creation of a supplier rating per competence.

- Decision Support System based Edition. For more complex announcements, some decision-making can be necessary. It can implicate the need for a kind of workflow to coordinate the execution of all intermediate steps (involving different people, different departments and different information sources) till a final decision is achieved.

\subsection{Suppliers Search}

In terms of the VE life cycle, this ACF matches the sub-activities 1.2 (seeking potential partners) and 2.1.1 (inclusion of a new member), identified in the Chapter 2. This ACF aims at providing an enterprise with tools to look for potential suppliers before spreading an announcement. This is a quite useful service in a VE platform since an enterprise normally works with many suppliers. This ACF can also be useful when making contacts with new suppliers. In a very «volatile» VE, in which its composition is made in a non-predefined way, the suppliers may be unknown a priori. On the other hand, even during the normal operation of a «stabilized»VE, it might be necessary to find suppliers for a particular component or service not offered by other VE members. This ACF can be also useful in the presence of unexpected events during the VE operation, for example, when a given VE member has problems in finishing / fulfilling some contracted business process. When this happens, a possible re-action of the VE Coordinator is to look for a substitute supplier, what can be supported by this ACF. This activity can be executed in some different ways, like searching in the enterprises' internal database, in «yellow pages» / services directories, in WWW homepages, in virtual business centers or even with help of a decision support system for a qualitative search. 


\subsection{Negotiation}

This ACF matches the sub-activity 1.3.1 (negotiation) as well as a support for the sub-activities 2.1 (VE Reconfiguration) and 2.2 (modification in the DBP contract), identified in the Chapter 2. During the formation of a VE some discrepancies between the VE coordinator requirements (represented in an announcement previously spread) and the interested suppliers can arise. Also, when a certain VE member, once a VE is in operation, has a problem and drops its BP out, it is very important to find a new partner in order to minimize the time to replace the problematic enterprise. These situations are quite normal to occur and it is usually solved via a negotiation process. Therefore, this ACF aims to provide an environment to support an electronic and semi-automatic negotiation in some stages of a whole negotiation process. There are many ways to proceed in a negotiation process until an agreement is reached. For example, applying some ideas issued from the Distributed Artificial Intelligence area. Usually, two partners could automatically negotiate with each other (with or without human supervision) looking for the relaxation of some values specified in an «announcement», such as those related with the product tolerance, due date, prices, etc. This activity can be executed using three cooperative modules:

- Sending and Receiving Messages. This class refers to all actions necessary to send and to receive messages related with an electronic and semi-automatic negotiation. It would correspond to the definition of a high-level negotiation protocol to support the interactions between two nodes of the network (Sandholm \&al., 1995). An interesting point is that the EDIFACT protocol can support many messages supposed to be necessary in a negotiaion situation. However, the most difficult problem is related with the control of interactions in a distributed decision-making environment. In a semi-automatic approach for negotiation, the user can play an important role to guide and to decide about some relaxing, for example. More than that, in some cases a decision cannot be taken by just one people / department, which implicates in a very tight control of all interactions and information flow. This aspect is strongly related with the other two classes of functionalities, next described.

- Decision Support for Announcement Modification. The evaluation about the impacts a modification / relaxing of some values in a given announcement can be complicated. This ACF class is a kind of decision support system (DSS) to aid a user in that evaluation during a negotiation process. This DSS could be a simulator of scenarios, similarly a MRP-II system, and would be able to show some results / information according to the modifications the user can perform on the scenarios. Some of the requirements for such a DSS includes the identification and modeling of the representative scenarios, the selection of the evaluation measures and the consideration of how distributed the user decision could be.

- Decision Support for Announcement Modification Implementation. This $\mathrm{ACF}$ can be seen as an auxiliary of the previous one. It is related with a set of 
services to provide means to control the modifications in a certain announcement under negotiation. The «implementation» of a modification may involve several different areas of the enterprise. It means the existence of a sequence of actions that each area can/may/must execute. For instance, sending a message to all involved departments in order to remind them to promote locally the required modifications by the end of a certain date.

\subsection{Edition and Contract Configuration}

In terms of the VE life cycle, this ACF matches the sub-activity 1.5 (legal agreement) as well as a support for the sub-activities 2.1 - VE Reconfiguration and 2.2 - modification in the DBP contract - identified in the Chapter 2. One of the problems to be faced in a VE is the effective («legal») contracting of the enterprises composing the VE (Scherer, 1997). That is, the macro-action that has to be carried out after the announcement and negotiation processes. In this sense, this ACF would be responsible to support an edition and contract configuration. This activity can be executed using two cooperative modules. Basically, they are:

- Contract Elaboration. It is related to a function able to support the actions necessary to elaborate a contract once the supplier is selected. The process to elaborate a contract normally differs from one enterprise to the other and from one product to the other. Besides that, the own meaning of «what a contract is» appears to be quite variable and it can be dependent of the knowledge the VE coordinator has about the supplier. For example, when necessary, a long document is prepared and configured for a new supplier. For other suppliers, a simple invoice print is enough. In fact, according to the end-users comments, the keyword is «trust». Inspite of these considerations, a contract can be elaborated based on «sketches» and/or historical contracts previously edited based on a «contract model». Some aspects that have to be considered in this model are the definition of who elaborate a contract, who negotiates its parameters, how are the penalties and how a task is considered "finished" so that an enterprise can apply or not the contract rules.

- Electronic Signing. Independently of the level of trust between two enterprises, a contract has to be signed at last. Instead of sending / receiving contracts by fax, telex or paper for further signature, the main objective of this $\mathrm{ACF}$ is to provide a network-based way able to provide a digital, reliable, secure and fast sending and receiving of signed contracts. It is known that nowadays the networks are still not completely secure from network «sniffers». Nevertheless, it is also known that there are a lot of work being done on it and this problem trends to get solved in short time. Another problem to be investigated is concerned with the legal aspects of transnational contracts. 


\subsection{Logistics Support}

This ACF acts as a support for the VE operation phase, identified in the Chapter 2. It aims to support means to get, to provide, and to manage the information from and about a VE, enabling the enterprises to make their logistics more efficiently. The basic motivation for this ACF is that the enterprises need reliable and timely information about the supply-chain and support for rapid decision-making and VE performance analysis. Hence accurate data for logistics is a must nowadays, it is one of the keys to improve the enterprise competitiveness A system for that can have three cooperative modules:

- VE Supervision. One of the main problems the enterprises face nowadays is the difficulty for obtaining reliable information from the partners about the real status of some contracted business processes, which directly affect the DBP. This situation obliges the enterprises to frequently re-plan their actions due to the occurrence of unexpected events representing on delays. It means an increase of the enterprise's global costs because a delay of one supplier can affect the execution of other inter-related business processes. This ACF aims to offer an electronic way to get information from the suppliers so that the enterprises can constantly update their production plans (Rabelo \&al., 1997).

- Decision-Support for Logistics Management. The introduction of a decision-support system (DSS) that helps an enterprise to evaluate and to decide in the presence of a conflict is a key point for its efficient reaction. This kind of system can play its role much better if it is fed with reliable and realtime information from the supply-chain, which is supposed to be provided by the functionality previously explained. It can incorporate empirical knowledge from the more experienced users as well as analytical tools as a support for its evaluations. For a complex scenario, such a DSS could have multi-paradigm tools in order to attend other kinds of situations in which a trade-off between optimality of results versus processing/reasoning time has to be carried out (Spinosa \& al., 1997). A DSS like that assumes a strong interaction with the enterprises' end-users.

- VE Analysis. This ACF is a very challenging one. Its objective is related to extend the classical MRP systems philosophy providing an enterprise with a more global view of the VE, allowing it to make a VE performance analysis. A tool like that would be much more efficient if it could receive updated information from the VE members and their current capacities, which is supposed to be provided by the VE supervision functionality. In this sense, the VE coordinator could try to optimize its requirements and capacity planning according to the workload (real or foreseen) of each VE member. Based on such optimization, the VE coordinator might try to review some aspects agreed in some contracts aiming at a more rational workload of the VE as a whole (Rabelo \&al., 1996). 


\section{IMPLEMENTATION ASPECTS AND FINAL REMARKS}

This paper presented a global approach to coordinate business processes in the virtual enterprise (VE). It corresponds to the development of high-level functionalities - the advanced coordination functionalities (ACFs) - that help an enterprise to deal with many activities within this scenario.

Covering the entire VE life cycle, a list of activities and subactivities were identified and proposed. Five main groups of ACFs emerged from that list. The ACFs, as software modules, are integrated in the Prodnet-II, a wider architecture that takes care of most part of the integration and interoperation problems. Each $\mathrm{ACF}$ has been introduced, explaning its utility and importance, as well as, some emerging aspects that have to be dealt in a VE context.

All software being developed has been implemented in PC/Windows-NT/C++ platform, specially devoted to the SMEs conditions. In the case of the ACFs, due to their natural variety (according to the VE domain), several implementation approaches can be used. Considering the ACFs tend to be heterogeneous, distributed, autonomous, requiring a high degree of intelligence and cooperation with each other and with the other modules, the object-oriented and multiagent (Bradshaw, 1997) approaches have strongly been investigated.

As a final remark, considering the implementation of ACFs, it is important to take into account the availability of many software in the Internet, which can be used to help solving very specific problems or even act as auxiliary for that. However, most of these packages are not open to be integrated at all.

\section{REFERENCES}

Bowersox, D.; Closs, D., (1996) - «Logistical Management: The Integrated Supply Chain Process», McGraw-Hill.

Bradshaw, J. (Ed.), (1997) - «Software Agents», AAAI Press / The MIT Press.

Camarinha-Matos, L. M.; C. Lima; Osório, L., (1997) - «The Prodnet plataform for production planning and management in virtual enterprises», Proceedings ICE'97 - $4^{\text {th }}$ International Conference on Concurrent Enterprising, pp. 385406, Nottingham UK.

Hunt, I.; Klen, A.; Zhang, J., (1997) - «Cross border enterprises: virtual and extended!», in Re-engineering for Sustainable Industrial Production, ", Ed. Luis M. Camarinha-Matos, Chapman \& Hall, pp. 63-72.

Rabelo, R.J.; Camarinha-Matos, L. M., (1994) - «Negotiation in Multiagent Based Dynamic Scheduling», Journal on Robotics and Computer Integrated Manufacturing, Vol 11 N 4, pp. 303-310, Pergamon.

Rabelo, R.J.; Camarinha-Matos, L. M., (1996) - «Towards agile scheduling in extended enterprise», in "Balanced Automation Systems II - Implementation challenges for anthropocentric manufacturing", Eds. Luis M. CamarinhaMatos and Hamideh Afsarmanesh, Chapman \& Hall, pp. 413-424. 
Rabelo, R.J.; Spinosa, L. M., (1997) - «Mobile-Agent-Based supervision in supply-chain management in the food industry», Proceedings Workshop on Supply-Chain Management in Agribusiness, Vitoria (ES) Brazil, pp. 451-460. Ross, D. F., (1996) - «Distribution Planning and Control», Chapman \& Hall. Sandholm, T.; Lesser, V., (1995) - «Issues in Automated Negotiation and Electronic Commerce: Extending the Contract Net Framework», Proceedings ICMAS'95 - First International Conference on Multiagent Systems, pp. 328325.

Scherer, R., (1997) - «Legal Framework for a Virtual Enterprise in Building Construction», Proceedings ICE'97 - $4^{\text {th }}$ International Conference on Concurrent Enterprising, pp. 373-384, Nottingham UK.

Spinosa, L. M.; Espinasse, B.; Chouraqui, E., (1997) - «For a Decision-Support System Model to Distributed Manufacturing Systems: a Multiagent and CIMOSA based Approach», Proceedings of MCPL'97 - IFAC/IFIP Conference on Management and Control of Production and Logistics, Campinas (SP) Brazil, pp. 504-509.

\section{BIOGRAPHIES}

Dr. Luiz Marcio Spinosa is a research scientist at the G-SIGMA at the Federal University of Santa Catarina (UFSC). He received his B.Sc. on Computer Science in 1986 and his M.Sc. in Mechanical Engineering in 1991 on CAD/CAM/CIM at UFSC. In 1992 he received his D.E.A on Automation \& Information Technology and in 1996 his Ph.D. on Production \& Information Technology at the University of Aix-Marseille III (France). His current research interests are: virtual enterprise, intelligent manufacturing systems, MAS and industrial modeling approaches.

Dr. Ricardo J. Rabelo is a research scientist at the G-SIGMA at the Federal University of Santa Catarina (UFSC). He received his B.Sc. on Computer Science in 1984 at UFSC / Brazil, worked as a consultant for several Brazilian companies and he finished in 1997 his Ph.D. Thesis at the New University of Lisbon (Portugal) on Robotics and CIM. His main interests are: agile scheduling, virtual enterprise, multiagent systems and information integration.

Dr Alexandra P. Klen Alexandra is a research scientist at the G-SIGMA in the Federal University of Santa Catarina (UFSC) in Brazil. She obtained a B.Sc. degree in Mechanical Engineering in 1988 from this university. She was an invited scientist at BIBA in Germany from April 1991 to September 1994. She obtained a $\mathrm{Ph} . \mathrm{D}$. degree for her work done in the area of 'An approach to Conceptualize Learning Enterprises in the Manufacturing Sector' in 1996 from UFSC. She currently works on ESPRIT project. 